\title{
Cross-Shaped Twisting Structure Using SMA-Based Smart Soft Composite
}

\author{
Hugo Rodrigue', Wei Wang', Binayak Bhandari', Min-Woo Han', and Sung-Hoon Ahn ${ }^{1,2 \sharp}$ \\ 1 Department of Mechanical and Aerospace Engineering, Seoul National University, Gwanak-Ro 566, Gwanak-gu, Seoul, South Korea, 151-742 \\ 2 Institute of Advanced Machinery and Design, Seoul National University, Gwanak-Ro 566, Gwanak-gu, Seoul, South Korea, 151-742 \\ \# Corresponding Author / E-mail: ahnsh@snu.ac.kr, TEL: +82-2-880-7110, FAX: +82-2-888-9073
}

\begin{abstract}
This paper introduces a novel geometry for a pure-twisting soft morphing actuator that improves the stability of the actuator and allows it to obtain a larger twisting angle. The smart soft composite (SSC) actuator uses pair of NiTi shape memory alloy (SMA) wires embedded in a cross-shaped polydimethylsiloxane (PDMS) matrix at constant and opposite eccentricity across the cross-section in opposite directions in order to produce a twisting motion. To evaluate the twisting performance of the cross-shaped actuator, specimens with rectangular cross-sections and cross-shaped cross-sections are made and their twist angles are measured and compared. Results show that the cross-shaped actuator is capable of a higher twisting rate by using a thinner flange due to a more stable twisting motion.
\end{abstract}

\section{Introduction}

Shape memory alloy (SMA) materials are capable of changing their shape and their mechanical properties due to their unique thermomechanical properties resulting from the shape memory effect inherent to this type of material. By mechanical loading, the material is brought from a twined martensite state to a detwined martensite, but is capable of recovering their shape when transforming to an austenite phase through thermal loading. The recoverable pre-strain of this type of material is about 4 to 5 percent of their initial length, but by embedding SMA elements within a soft matrix it is possible to produce a continuous large deformation of the matrix. ${ }^{1}$

Soft morphing actuators do not need any type of external source of actuation and are thus a good choice as a compact and lightweight actuator in critical applications. They have been used in morphing applications such as morphing wings ${ }^{2}$ in order to increase performance, control and fuel efficiency of unmanned aerial vehicles (UAV). However, limitations exist in terms of performances and modes of actuation.

This type of actuator was first proposed in 1992 by Lagoudas and Tadjbakhsh where SMA fibers were embedded in a flexible rod. ${ }^{3}$ Bending actuators with embedded SMA elements have been proposed by Icardi, ${ }^{4}$ Wang and Shahinpoor, ${ }^{5}$ Sun and Sun $^{6}$ and Choi and Lee. ${ }^{7}$ Wang et al. ${ }^{8}$ and Zhou and Lloyd ${ }^{9}$ showed that a softer matrix leads to larger deflection while the latter also showed that a thinner matrix leads to a higher deflection. Ryu et al. ${ }^{10}$ used alternated harder and softer sections to increase the deflection of the actuator. Jung et al. ${ }^{11}$ showed that a thinner structure along with higher SMA eccentricity leads to a higher deflection of the actuator.

Spinella et al. ${ }^{12}$ and Paik et al. ${ }^{13}$ developed twisting actuators with externally embedded SMA elements. However, Kim et al. ${ }^{14}$ were the first to realize a large twisting deformation of a structure through embedded SMA wire, but this twisting motion was coupled to a bending deflection.

In this work, a smart soft composite (SSC) twisting actuator with a cross-shaped cross-section is presented and compared to rectangularshaped actuator with the same actuation mechanism. The cross-shaped cross-section allows the use of a thinner matrix which leads to a higher twisting angle. The design, fabrication method, model and results of the rectangular shaped actuator and a cross-shaped actuator are presented and compared.

\section{Actuator Design and Experimental Set Up}

This actuator functions by having two SMA wires at an angle within the matrix at opposite eccentricity which create a twisting force around a center of rotation and negate the bending moment created by using 


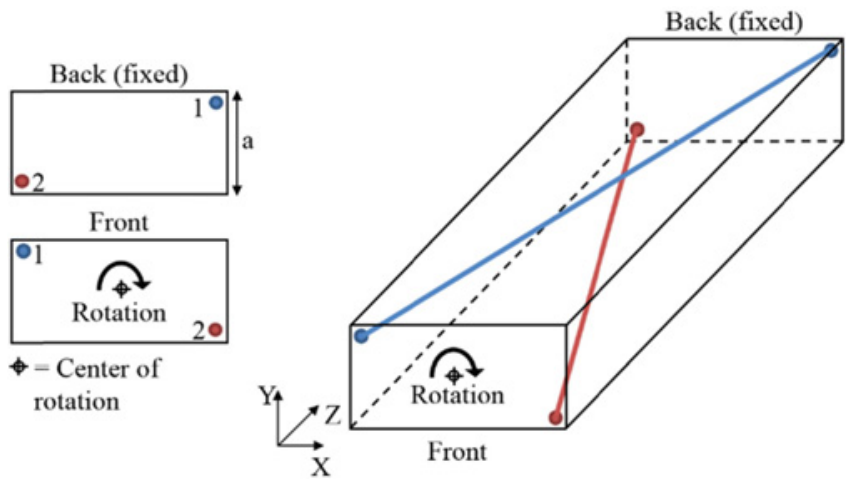

Fig. 1 Configuration of the rectangular twisting actuator

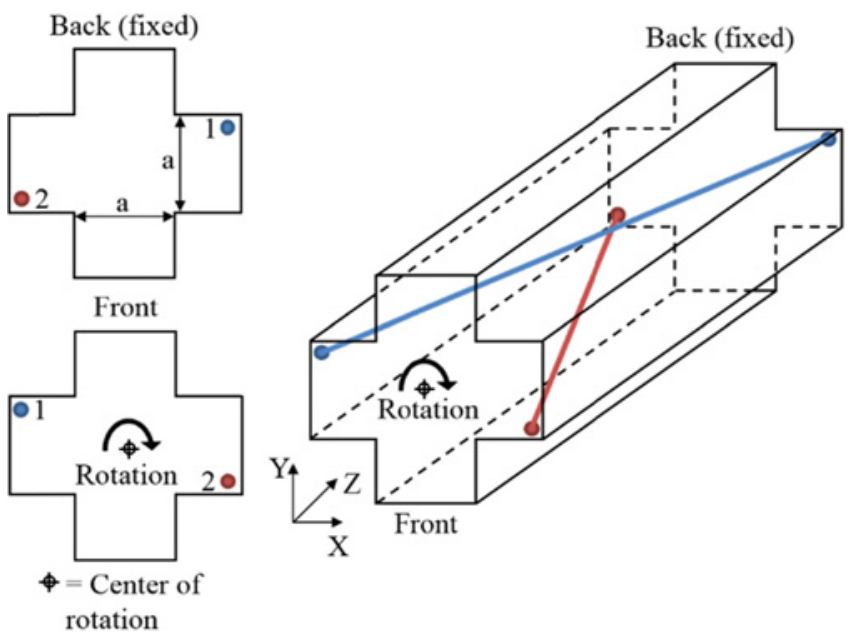

Fig. 2 Configuration of the cross-shaped twisting actuator

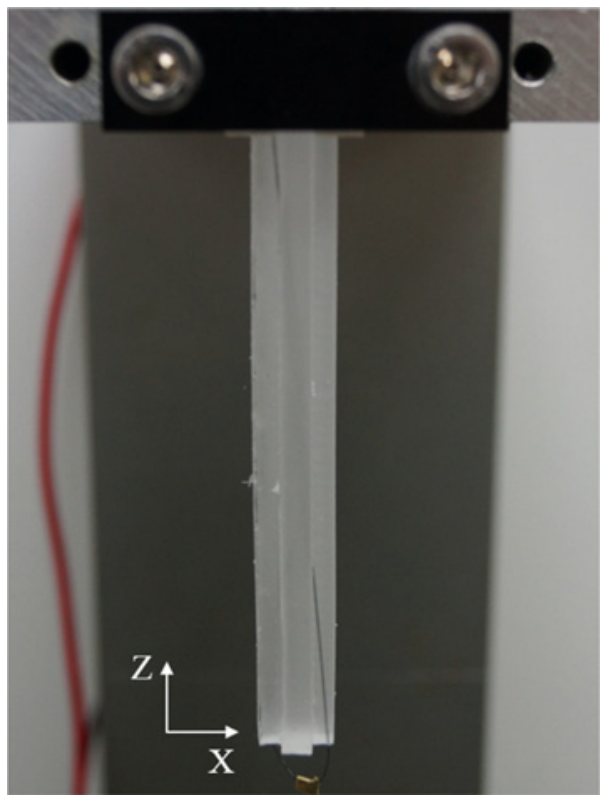

Fig. 3 Experimental setup for the twisting angle

a single SMA wire. Two types of actuators are used in this paper, the first is with a rectangular cross-section and the second is with a crossshaped cross-section. Fig. 1 shows the configuration of the rectangular actuator and Fig. 2 shows the cross-shaped actuator with the dimensional variable being labeled as "a" in both figures. Molds are manufactured by pouring polydimethylsiloxane (PDMS) in an acrylonitrile butadiene styrene (ABS) mold manufactured by rapid prototyping (RP) with holes used to position the SMA wires (FLEXINOL from Dynalloy, Inc., NiTi) which are pre-strained and fixed. The mold is removed after curing of the assembly at a temperature below the martensite transformation temperature.

Throughout this paper, the width will be referred as being in the $\mathrm{X}$ direction, the thickness as being in the $\mathrm{Y}$ direction and the length as being in the $\mathrm{Z}$ direction.

The actuator is fixed at one end with the length being in the vertical position, the actuator is actuated through joule heating of the SMA wires until no further movement can be detected visually. The twisting angle is then measured by taking the angle between the fixed end and the free end. The experimental setup is shown in Fig. 3, where the twisting angle is measured about the $\mathrm{Z}$ axis.

\section{Model}

Equation 1 is used to predict the twisting angle of each actuator where $\theta$ is the twisting angle, $T$ is the twisting force, $L$ is the length of the actuator, $J$ is the torsion constant and $G$ is the shear modulus of PDMS (0.61 MPa).

$$
\theta=\frac{T L}{J G}
$$

$T$ is calculated using Equation 2 which calculates the twisting force produced by the components of the forces in $\mathrm{X}$ of both SMA wires about the center of rotation. $l_{\text {mat }}$ and $w_{\text {mat }}$ are respectively the length and width of the matrix, $t_{S M A}$ is the distance in Y between the two SMA wires and $F_{S M A}$ is the maximum pull force of the SMA wire $(5.586 \mathrm{~N})$ as described by the manufacturer. ${ }^{15}$

$$
M=F_{S M A} t_{S M A} \cos \left(\tan ^{-1} \frac{l_{\text {mat }}}{W_{\text {mat }}}\right)
$$

$J$ is calculated using Equation 3 for the rectangular actuator where $t_{m a t}$ is the thickness of the matrix and using Equation 4 for the crossshaped actuator where $t_{\text {flange }}$ is the thickness of the flanges.

$$
\begin{gathered}
J_{\text {rect }}=t_{\text {mat }} w_{\text {mat }}^{3}\left(\frac{1}{3}-0.21 \frac{t_{\text {mat }}}{w_{\text {mat }}}\left(1-\frac{w_{\text {mat }}^{4}}{12 t_{\text {mat }}^{4}}\right)\right) \\
J_{\text {cross }}=\left(\frac{w_{\text {mat }}}{t_{\text {flange }}}-0.373\right) \frac{t_{\text {flange }}^{4}}{3}
\end{gathered}
$$

\section{Results}

Rectangular actuators with a length and width of $100.0 \mathrm{~mm}$ by $10.0 \mathrm{~mm}$ were built with varying thicknesses. Three samples were built for each thickness of $10.0 \mathrm{~mm}, 8.0 \mathrm{~mm}, 6.0 \mathrm{~mm}, 4.0 \mathrm{~mm}, 3.0 \mathrm{~mm}$, $2.5 \mathrm{~mm}, 2.0 \mathrm{~mm}$ and $1.5 \mathrm{~mm}$. Due to the thin matrix, the actuators with thicknesses of $1.5 \mathrm{~mm}$ and $2.0 \mathrm{~mm}$ produced too much bending to have a measurable twisting angle. Results for the other samples are shown in Fig. 4 with the results of the model shown as a dashed line.

Results show that the twisting angle is much lower than that predicted 


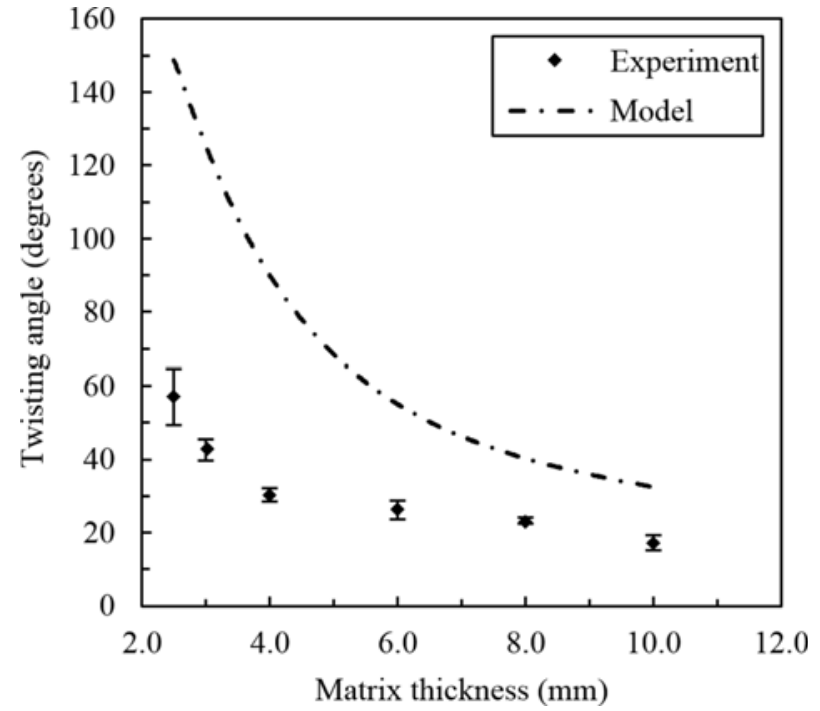

Fig. 4 Twisting angles for rectangular actuators with different matrix thicknesses

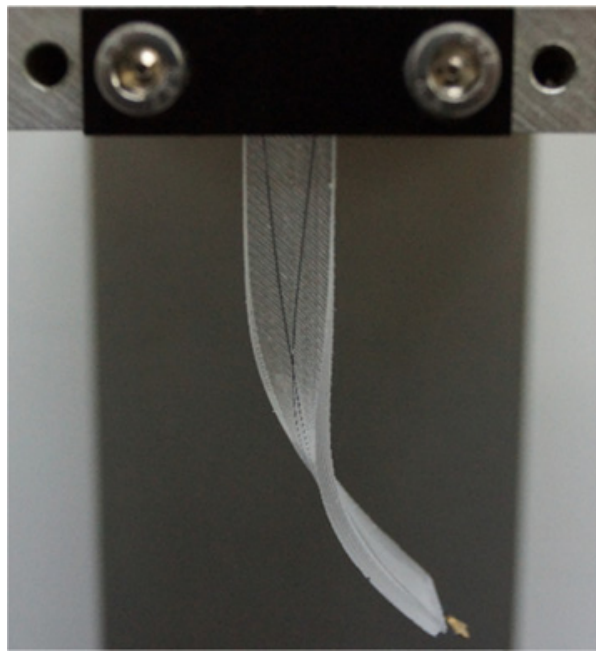

Fig. 5 Failure of a rectangular actuator with a $2 \mathrm{~mm}$ thickness due to bending of the actuator

by the model. This is due to bending of the matrix during actuation which reduces the twisting angle. As the matrix gets thinner, larger bending of the matrix is observed and bending becomes the main mode of actuation in the case of the actuators with matrix thicknesses of $2.0 \mathrm{~mm}$ and $1.5 \mathrm{~mm}$. This is due to the force of the SMA acting in the $\mathrm{Z}$ direction of the actuator being too large for the actuator to sustain due to a low moment of inertia $\left(I_{Y Y}\right)$ in the $\mathrm{Y}$ direction. This results in bending of the actuator as shown in Fig. 5.

Actuators with a cross-shaped cross-section with a length of $100.0 \mathrm{~mm}$, overall cross-section dimensions of $10.0 \mathrm{~mm}$ by $10.0 \mathrm{~mm}$ and variable flange thicknesses were built. The thickness of all four flanges are equal in order to obtain $I_{X X}=I_{Y Y}$. Samples were built with flange sizes of $4.0 \mathrm{~mm}, 3.5 \mathrm{~mm}, 3.0 \mathrm{~mm}, 2.5 \mathrm{~mm}, 2.0 \mathrm{~mm}$ and $1.5 \mathrm{~mm}$. Results are shown in Fig. 6 with the results of the model shown as a dashed line.

Results show that the actuator was capable of producing a stable twisting motion for all flange thicknesses with the maximum twisting angle being produced by the actuator with a flange thickness of $2.0 \mathrm{~mm}$ with $1.5 \mathrm{~mm}$ producing a similar twisting angle. At lower flange

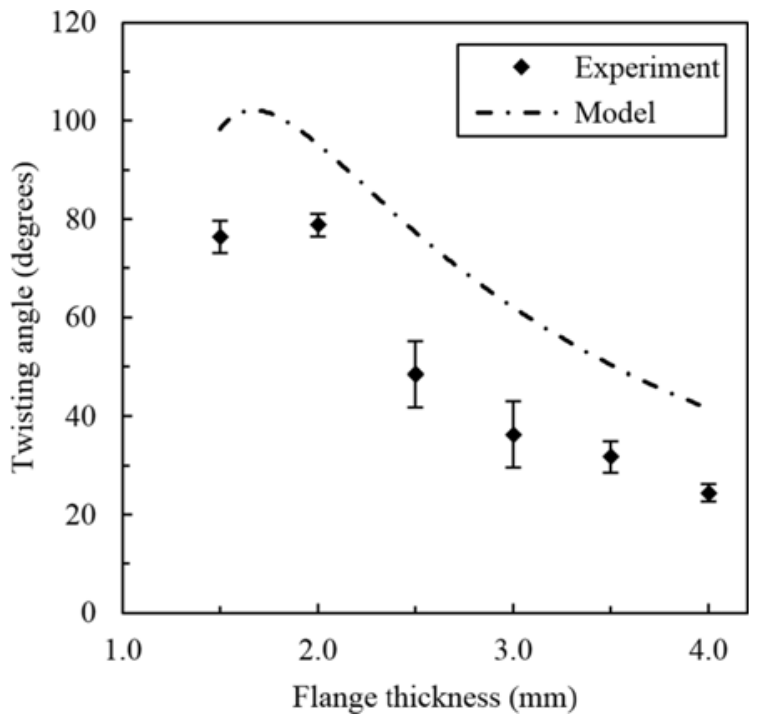

Fig. 6 Twisting angles for cross-shaped actuators with different flange thicknesses

thicknesses, the reduction in torsion constant $J$ is balanced by the reduction in distance between the SMA wires $t_{S M A}$, resulting in a reduction of the twisting angle.

When the matrix thickness of the rectangular actuator and the flange thickness of the cross-shaped actuator are equal, the torsion constant $(J)$ is higher for the cross-shaped actuator. However, the bending of the matrix of the rectangular actuator diminishes its performance such that the twisting angle is similar for both actuators with the same flange and matrix thickness. Furthermore, the higher stability resulting from having $I_{X X}=I_{Y Y}$ allows for a thinner flange thickness than that allowable for a rectangular actuator of the same thickness. This results in a larger maximum possible twisting angle for a cross-shaped actuator of a certain length and width than that of a rectangular actuator with the same length and width. Thus, the maximum angle of the cross-shaped actuator was 81.05 degrees versus 56.4 degrees for the rectangular actuator.

\section{Conclusion}

In this work, a novel geometry for a pure-twisting soft morphing actuator was developed in order to obtain a larger twisting angle. This geometry increases the stability of the actuator which allows for a thinner matrix resulting in a larger twisting angle. Samples were built for a rectangular cross-section and a cross-shaped cross-section with varying geometry thicknesses. Results showed that the rectangular shaped actuator failed when the matrix was too thin due to large bending of the actuator. However, the cross-shaped actuator was able to remain stable for flange thicknesses as thin as $1.5 \mathrm{~mm}$. Thus, the maximum angle of the cross-shaped actuator was 43 percent higher than that of the rectangular shaped actuator for the width and length used in this experiment.

\section{ACKNOWLEDGEMENT}

This research was supported by the Converging Research Center Program through the Ministry of Science, ICT, and Future Planning, 
Korea (No. 2013K000371), the National Research Foundation of Korea(NRF) grant funded by the Korea government (MEST) (NRF2010-0029227), the Brain Korea 21 Plus Project in 2014 and by a grant to Bio-Mimetic Robot Research Center funded by Defense Acquisition Program Administration (UD130070ID).

\section{REFERENCES}

1. Ahn, S. H., Lee, K. T., Kim, H. J., Wu, R., Kim, J. S., and Song, S. H., "Smart Soft Composite: An Integrated 3D Soft Morphing Structure using Bend-Twist Coupling of Anisotropic Materials," Int. J. Precis. Eng. Manuf., Vol. 13, No. 4, pp. 631-634, 2012.

2. Barbarino, S., Bilgen, O., Ajaj, R. M., Friswell, M. I., and Inman, D. J., "A Review of Morphing Aircraft," J. Intell. Mater. Syst. Struct., Vol. 22, No. 9, pp. 823-877, 2011.

3. Lagoudas, D. and Tadjbakhsh, I., "Active Flexible Rods with Embedded SMA Fibers," Smart Mater. Struct., Vol. 1, No. 2, pp. 162-167, 1992.

4. Icardi, U., "Large Bending Actuator Made with SMA Contractile Wires: Theory, Numerical Simulation and Experiments," Compos. Part B-Eng., Vol. 32, No. 3, pp. 259-267, 2001.

5. Wang, G. and Shahinpoor, M., "Design, Prototyping and Computer Simulations of a Novel Large Bending Actuator Made with a Shape Memory Alloy Contractile Wire," Smart Mater. Struct., Vol. 6, No. 2, pp. 214-221, 1997.

6. Sun, G. and Sun, C. T., "Bending of Shape-Memory AlloyReinforced Composite Beam,” J. Mater. Sci., Vol. 30, No. 22, pp. 5750-5754, 1995.

7. Choi, S. and Lee, J. J., "The Shape Control of a Composite Beam with Embedded Shape Memory Alloy Wire Actuators," Smart Mater. Struct., Vol. 7, No. 6, pp. 759-770, 1998.

8. Wang, Z., Hang, G., Wang, Y., Li, J., and Du, W., "Embedded SMA Wire Actuated Biomimetic Fin: a Module for Biomimetic Underwater Propulsion," Smart Mater. Struct., Vol. 17, No. 2, Paper No. 025039, 2008.

9. Zhou, G. and Lloyd, P., "Design, Manufacture and Evaluation of Bending Behaviour of Composite Beams Embedded with SMA wires," Compos. Sci. Technol., Vol. 69, No. 13, pp. 2034-2041, 2009.

10. Ryu, J. H., Jung, B. S., Kim, M. S., Kong, J. P., Cho, M. H., and Ahn, S. H., "Numerical Simulation of Hybrid Composite ShapeMemory Alloy Wire-Embedded Structures," J. Intell. Mater. Syst. Struct., Vol. 22, No. 17, pp. 1941-1948, 2011.

11. Jung, B. S., Kong, J. P., Li, N. X., Kim, Y. M., Kim, M. S., Ahn, S. H., and Cho, M. H., "Numerical Simulation and Verification of a Curved Morphing Composite Structure with Embedded Shape Memory Alloy Wire Actuators," J. Intell. Mater. Syst. Struct., Vol. 24, No. 1, pp. 89-98, 2012.
12. Spinella, I., Scirè Mammano, G., and Dragoni, E., "Conceptual Design and Simulation of a Compact Shape Memory Actuator for Rotary Motion,” J. Mater. Eng. Perform., Vol. 18, No. 5-6, pp. 638648, 2009.

13. Paik, J. K., Hawkes, E., and Wood, R. J., "A Novel Low-Profile Shape Memory Alloy Torsional Actuator," Smart Mater. Struct., Vol. 19, No. 12, Paper No. 125014, 2010.

14. Kim, H. J., Song, S. H., and Ahn, S. H., "A Turtle-Like Swimming Robot using a Smart Soft Composite (SSC) Structure," Smart Mater. Struct., Vol. 22, No. 1, Paper No. 014007, 2013.

15. Dynalloy, Inc., "FLEXINOL $®$ Actuator Wire Technical and Design Data," http://www.dynalloy.com/TechDataWire.php.html (Accessed 30 Oct. 2013) 